Recepción: 20 / 04 / 2017

Aceptación: 20 / 05 / 2017

Publicación: 15 / 06 / 2017

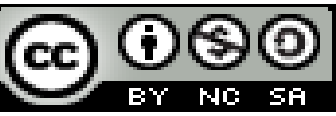

Ciencias Sociales

Artículo de Investigación

\title{
La inmediación y la concentración como principios constitucionales en la
}

legislación ecuatoriana

\author{
Immediacy and concentration as constitutional principles in \\ Ecuadorian legislation \\ Imediação e concentração como princípios constitucionais na \\ legislação equatoriana
}

Gissela A. Cevallos-Sánchez

gissela.cevalloss@ug.edu.ec

Zoila F. Alvarado-Moncada " zoila.alvaradom@ug.edu.ec

Washington R. Astudillo-Orellana "' washington.astudilloo@ug.edu.ec

Correspondencia: gissela.cevalloss@ug.edu.ec

I. Magister en Derecho Civil y Procesal Civil; Abogada de los Tribunales y Juzgados de la República del Ecuador; Licenciado en Mercadotecnia; Universidad de Guayaquil, Guayaquil, Ecuador.

II. Magister en Ciencias Penales y Criminológicas; Magister en Derechos Fundamentales y Justicia Constitucional; Especialista en Procedimientos Constitucionales; Diploma Superior en Derecho Constitucional y Derecho Fundamentales; Especialista en Ciencias Penales y Criminológicas; Licenciada en Ciencias Sociales y Politices; Abogado de los Tribunales y Juzgados de la República del Ecuador; Doctora en Jurisprudencia; Universidad de

III. Guayaquil, Guayaquil, Ecuador. Constitucional; Diploma Superior en Criminalística; Especialista en Procedimientos Constitucionales; Diploma Superior en Docencia Universitaria; Magister en Ciencias Penales y Criminológicas; Diploma Superior en Derecho Constitucional y Derechos Fundamentales; Especialista en Ciencias Penales y Criminológicas; Doctor en Jurisprudencia; Licenciado en Ciencias Sociales y Políticas; Abogado de los Tribunales y Juzgados de la República del Ecuador; Universidad Guayaquil, Guayaquil, Ecuador. 
Gissela A. Cevallos-Sánchez; Zoila F. Alvarado-Moncada; Rene W. Astudillo-Orellana

\section{Resumen}

El tema que nos ocupa en este espacio aborda la problemática de la ejecución de los Principios Constitucionales del Debido Proceso como Garantía Fundamental en el Ecuador; se esbozan conceptos del derecho a la tutela judicial efectiva, y se expone la necesidad de los cambios, que requiere el sistema judicial, no solamente a nivel del sistema de administración de justicia; sino también en la conceptualización misma del proceso como medio para proteger adecuadamente los derechos de las personas.

Se analizan las principales características y su relación con el principio de inmediación y concentración en el proceso oral civil, como consecuencia de las distintas causas que congestionan el sistema procesal civil en nuestro país, y que por ende no dejan que se cumpla con la garantía constitucional de la tutela efectiva de la justicia con celeridad y economía procesal a la que tenemos derecho todas las personas.

Por ello ésta investigación tiene como objetivo establecer los efectos jurídicos de la implementación efectiva del principio de inmediación y concentración en el procedimiento oral civil ecuatoriano, con miras a cumplir con las garantías del marco del buen vivir.

Palabras clave: Inmediación; tutela; concentración; oralidad; proceso; principios. 


\begin{abstract}
The subject that concerns us in this space deals with the problems Key words: immediacy, guardianship, concentration, orality, process, principles. Key words: immediacy, guardianship, concentration, orality, process, principles.of the implementation of the constitutional principles of due process as a Fundamental guarantee in the Ecuador; outlines concepts of the right to effective judicial protection, and exposed the need for changes, which requires the judicial system, not only at the level of the system of administration of Justice; but also in the same conceptualization of the process as a means to adequately protect the rights of the people. Discusses the main features and their relationship with the principle of immediacy and concentration in the oral civil proceedings, as a result of the different causes that congested the civil litigation system in our country, and therefore do not let compliance with the constitutional guarantee of effective protection of Justice quickly and procedural economy to which we have the right persons. Therefore this research is intended to establish the legal effects of the effective of the principle of immediacy and concentration implementation in the Ecuadorian civil oral procedure, to comply with the guarantees of the framework of the good living.
\end{abstract}

Key words: immediacy, guardianship, concentration, orality, process, principles. 
Gissela A. Cevallos-Sánchez; Zoila F. Alvarado-Moncada; Rene W. Astudillo-Orellana

\section{Resumo}

$\mathrm{O}$ assunto que nos interessa neste espaço trata dos problemas. Palavras-chave: imediatismo, tutela, concentração, oralidade, processo, princípios. Palavras-chave: imediatismo, tutela, concentração, oralidade, processo, princípios. Da implementação dos princípios constitucionais do devido processo como garantia fundamental no Equador; Descreve conceitos do direito a uma proteção judicial efetiva e expõe a necessidade de mudanças, que requer o sistema judicial, não só ao nível do sistema de administração da Justiça; Mas também na mesma conceptualização do processo como um meio para proteger adequadamente os direitos das pessoas. Discute as principais características e seu relacionamento com o princípio do imediatismo e da concentração no processo civil oral, como resultado das diferentes causas que congestionaram o sistema de litígio civil em nosso país e, portanto, não permitem o cumprimento da garantia constitucional de proteção efetiva De Justiça, economia rápida e processual à qual temos as pessoas certas. Por conseguinte, esta pesquisa destina- se a estabelecer os efeitos jurídicos do efetivo do princípio da implementação imediata e de concentração na modalidade oral civil equatoriana, para cumprir as garantias do quadro da boa vida.

Palavras chave: Imediatismo, tutela, concentração, oralidade, processo, princípios. 


\section{Introducción.}

El tema La Inmediación y Concentración como principios constitucionales, se desarrolla como parte fundamental del estudio de los Principios Constitucionales en la Legislación ecuatoriana, y se analiza el principio de concentración y la inmediación como elementos de la implementación del principio de oralidad en el sistema de justicia ecuatoriano. Para una mejor comprensión de los principios se efectúa una investigación de los diferentes conceptos que nos expone la doctrina en función de la concentración e inmediación como principios, y las características que rigen a los mismos.

Se aborda en este trabajo el tema de la inmediación y las facultades atribuidas al juez en el nuevo proceso en materias no penales; además se detalla la importancia del estudio de los principios de inmediación y concentración en el nuevo sistema procesal civil oral, ecuatoriano.

Finalmente llegamos a la presentación de la conclusión del trabajo de investigación, tomando en consideración que la implementación adecuada de los principios de tutela judicial efectiva, inmediación, concentración y oralidad en el sistema de justicia ecuatoriano, permitirá la consecución de la agilidad en los trámites procesales civiles, lo que garantiza al ciudadano la seguridad jurídica, con el fin de alcanzar, a través de la justicia el tan anhelado derecho al buen vivir.

La concentración y la inmediación como elemento de la oralidad.

En éste espacio analizaremos de manera sucinta la importancia de la necesidad de la implementación efectiva del principio de oralidad, inmediación y concentración en la tramitación de los procesos, en éste caso en materia civil, porque decimos en éste caso, porque si bien es cierto, los principios del debido proceso se los han utilizado desde hace mucho tiempo atrás, es decir, en el 
proceso penal, pero se ha llegado a estimar que si hablamos de proceso en materia civil también se deben implementar, porque existen juicios que requieren de la agilidad en los trámites a ejecutarse, bien conocemos que los distintos procesos que se recogen en materia civil, deben evacuarse en audiencias orales, para evitar de esta forma el papeleo absurdo, que genera desgaste de las partes y de los operadores de justicia.

La norma constitucional dispone lo siguiente: "La sustanciación de los procesos en todas las materias, instancias, etapas y diligencias se llevará a cabo mediante el sistema oral, de acuerdo con los principios de concentración, contradicción y dispositivo", según ésta disposición constitucional se debe entender que todos los procesos y las diligencias que ellos conllevan deberán ser ejecutados de manera oral, dejando atrás el sistema escrituitario que perduró por muchos años en nuestro sistema judicial y que sin dudarlo nos atrevemos a decir que es una de las causas que ocasiona la lentitud procesal en el sistema de justicia, la implementación efectiva de los principios de inmediación y concentración, permiten que el juez tenga un contacto directo con las partes y que las pruebas que se ejecuten sean en presencia del mismo, permitiéndole a este una visión real de los hechos.

\section{Materiales y métodos.}

La concentración y la inmediación como elemento de la oralidad.

En éste espacio analizaremos de manera sucinta la importancia de la necesidad de la implementación efectiva del principio de oralidad, inmediación y concentración en la tramitación de los procesos, en éste caso en materia civil, porque decimos en éste caso, porque si bien es cierto, los principios del debido proceso se los han utilizado desde hace mucho tiempo atrás, es decir, en el 
proceso penal, pero se ha llegado a estimar que si hablamos de proceso en materia civil también se deben implementar, porque existen juicios que requieren de la agilidad en los trámites a ejecutarse, bien conocemos que los distintos procesos que se recogen en materia civil, deben evacuarse en audiencias orales, para evitar de esta forma el papeleo absurdo, que genera desgaste de las partes y de los operadores de justicia.

La norma constitucional dispone lo siguiente: "La sustanciación de los procesos en todas las materias, instancias, etapas y diligencias se llevará a cabo mediante el sistema oral, de acuerdo con los principios de concentración, contradicción y dispositivo”, según ésta disposición constitucional se debe entender que todos los procesos y las diligencias que ellos conllevan deberán ser ejecutados de manera oral, dejando atrás el sistema escrituitario que perduró por muchos años en nuestro sistema judicial y que sin dudarlo nos atrevemos a decir que es una de las causas que ocasiona la lentitud procesal en el sistema de justicia, la implementación efectiva de los principios de inmediación y concentración, permiten que el juez tenga un contacto directo con las partes y que las pruebas que se ejecuten sean en presencia del mismo, permitiéndole a este una visión real de los hechos.

\section{Concepto de Concentración.}

Como hemos citado anteriormente entendemos por Concentración (Cabanellas de las Cuevas, 2014) "Al acto y consecuencia de concentrar o de concentrarse, este concepto hace referencia al logro de reunir en un determinado punto lo que se encontraba separado", es decir, para nuestro estudio entendemos que la concentración en el proceso nos permite reducir, en menos actos todas las piezas procesales de manera que se pueda dar agilidad a las causas en el menor tiempo posible, garantizado la celeridad y economía procesal. 
Gissela A. Cevallos-Sánchez; Zoila F. Alvarado-Moncada; Rene W. Astudillo-Orellana

Concepto de Inmediación.

Por inmediación, en materia de derecho entendemos que es un principio constitucional del derecho procesal, que está orientado a la relación directa de las partes litigantes con el juez, prescindiendo de la intervención de otras personas.

En nuestra Constitución de la Republica, éste principio es de gran importancia porque dentro del proceso, se encuentra directamente ligado al principio de la oralidad, ya que a través de él, se facilita la relación de las partes con el juez, puesto que éste escucha, in situ, a todas las partes en igualdad de condiciones, lo que le permite recrear un panorama más real de los hechos que debe juzgar por sí mismo, sin la intervención de ningún otro elemento que incida en su decisión.

A continuación, presentamos un gráfico que ilustra los principios constitucionales en el nuevo proceso oral civil ecuatoriano, a partir de las disposiciones constitucionales y del Código Orgánico de la Función Judicial del Ecuador: 


\section{Gráfico N¹}

\section{Principios constitucionales en el nuevo proceso oral civil ecuatoriano.}

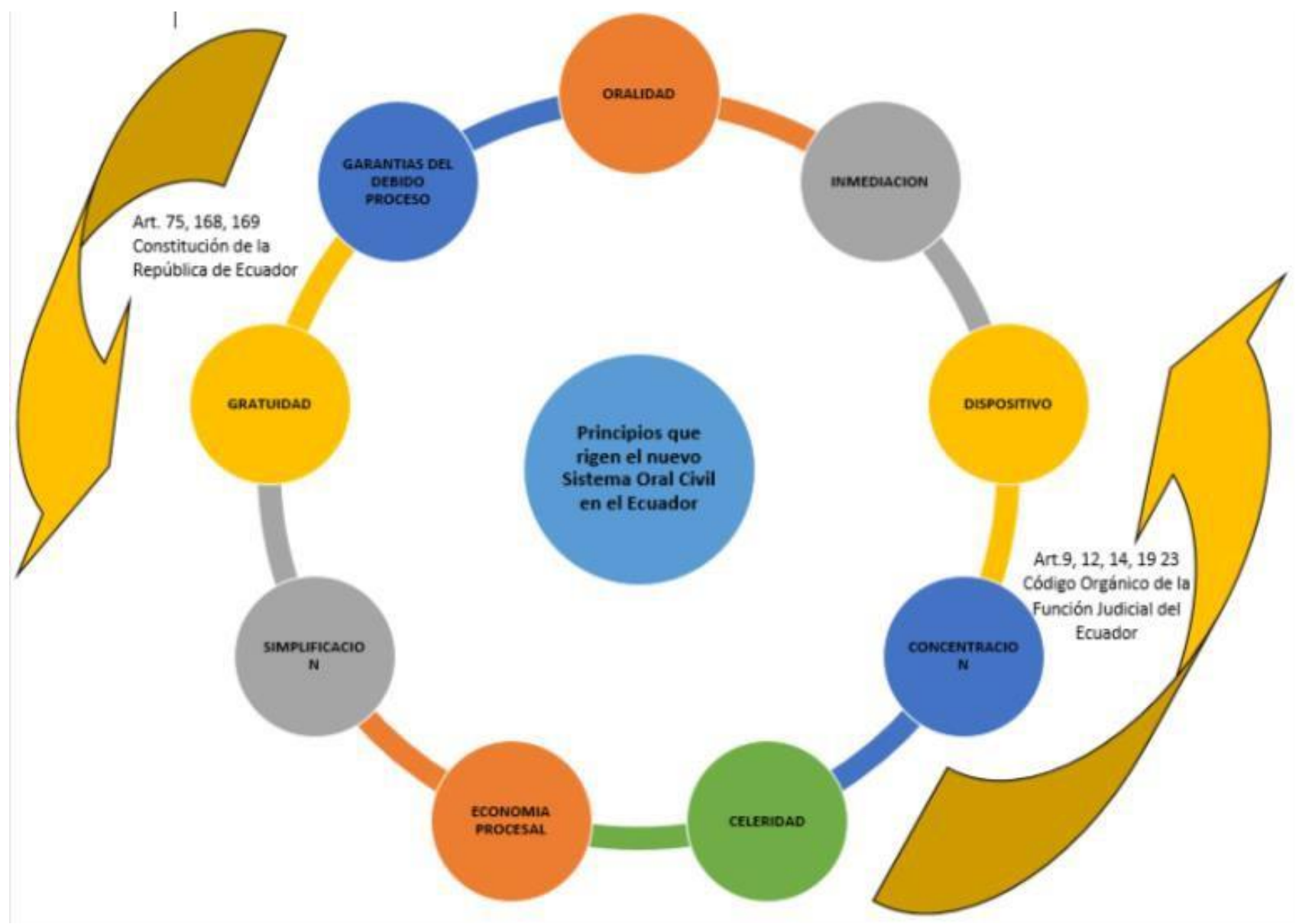

Fuente: Constitución de la República del Ecuador. Código Orgánico de la Función Judicial del Ecuador.

\section{Características que rigen los principios de concentración e inmediación.}

En el estudio de los principios de inmediación y concentración, para nuestra investigación, es necesario que se determinen sus características, por lo cual hemos llegado a establecer que según José Martín Ostos. (2012). Recuperado el 10 de octubre del 2014 de http://www.derechoprocesal.es/2012/10/principio-de-concentracion.html, tenemos las siguientes características: 
Su relación con la oralidad; difícilmente un procedimiento escrito permite la concentración de actuaciones, pues hay que esperar a la presentación de un escrito para, tras su detenido examen, preparar la adecuada contestación también escrita, dentro de un plazo, y así sucesivamente.

La conveniencia de la concentración para asuntos no excesivamente complejos (por ejemplo, en el actual juicio verbal civil, no en el ordinario en el que se ventila otro tipo de asuntos).

Al analizar estas características comprendemos que necesariamente la concentración de las causas, hace viable la oralidad en el proceso, puesto que si nos detenemos a examinar, nuestro proceso civil que tradicionalmente ha sido escrito, no permite la concentración, ya que su tramitología (exceso de papeleo), hace que se deba esperar un tiempo determinado para la debida contestación, lo que origina el retardo de las causas, que ya conocemos.

Mientras que en la inmediación encontramos que Liliana Romero (2012). Recuperado el 10 de octubre del 2014 de http://investigaciondoctrinaria.blogspot.com/2013/06/el-principio-deinmediacion-procesal.html nos señala las siguientes características que la identifican:

La presencia física del juez,

La recepción de alegatos y pruebas durante la audiencia,

El juez que falla es quien ha presenciado la audiencia.

Estas características destacan la importancia del principio de inmediación dentro del proceso, como vemos permite el acercamiento del juez y las partes, desde su inicio. Lo que conlleva a que el magistrado se familiarice con las causas, de manera que pueda efectuar un análisis más real de la situación, lo que hará que al dar su sentencia ésta sea realmente justa, porque es el mismo juez que participó de la audiencia, quien emite el fallo final. 


\section{La inmediación y las facultades del juez.}

Al efectuar el estudio de las facultades que tiene el juez, dentro del proceso civil, debemos comenzar por dejar sentado que el proceso civil ecuatoriano estaba inmerso en el caos imperante de la corrupción; y, por lo tanto en este modelo de sistema el juez, no poseía las mismas facultades de las goza hoy en día, vale destacar que no con ello se justifica el accionar ambiguo que operó por años en los fallos judiciales.

De tal manera debemos entender que gracias al principio de inmediación en el proceso, el juez va a tener un número mayor de facultades dentro de este, lo que le permite tener un contacto directo, con todos las partes que lo conforman, de ésta forma su aproximación con ellas, le va a permitir formarse mejores elementos de convicción, para expedir un fallo que no conculque los derechos de quien acude a él en pos de justicia. Citamos la obra de (Rioja Bermudez, 2011): “Al optar por la inmediación, el código, ha privilegiado también la oralidad, el medio por el cual se produce el contacto directo entre el Juez y los protagonistas directos o indirectos del proceso", considerando la valía de su aporte en el derecho comparado, para el nuevo proceso civil oral en el Ecuador.

Los jueces son quienes por el poder que les otorga el Estado, están facultados para impartir justicia en nombre de él, por ello es importante destacar que su rol ante la sociedad, requiere de su compromiso para con los ciudadanos ávidos del cambio que sé de a la justicia, para hallar la verdad de los hechos. Es imperiosa la necesidad de que los operadores de justicia hagan conciencia en el papel que tienen frente a la sociedad.

Fundamentándonos en las líneas que anteceden citamos el siguiente texto de (Zavala Baquerizo, 2001) quien dice: "La labor del juez vas mucho más allá de la aplicación de la Ley y de la propia Constitución, es creativa". Si analizamos éste enunciado, veremos que efectivamente el 
juez debe ser más que un juzgador, y convertirse en un jurista creativo, que fundamentado en la ley busque vías alternas de resolución de conflictos, procurando de esta forma que impere la justicia expedita.

\section{Importancia de la inmediación y la concentración en el nuevo sistema procesal civil oral}

Al determinar la importancia de los principios de inmediación y concentración en el nuevo proceso en materias no penales, es preciso, explicar porque nos referimos a un proceso oral nuevo, y esto lo manifestamos tomando en consideración que a pesar, de que ya en la Constitución del año 1998, se disponía la oralidad en los procesos, esta disposición no se cumplía, por varias causas; sin embargo en la actualidad ésta disposición cobra relativa importancia en primer lugar en el cambio que se dé en relación a la administración de justicia por parte de quienes la conforman, ya que de nada sirve el contar con una estructura material, sino se cuenta con el talento humano idóneo para impartir justicia.

Se han efectuado grandes cambios en el sistema judicial de nuestro país, estamos conscientes que las autoridades de turno se han preocupado por ejecutar políticas públicas destinadas a mejorar la calidad de los servicios en el sistema judicial; sin embargo no es menos conocido que los esfuerzos realizados, aún no ven sus frutos, por cuanto en el sistema de justicia aún persiste la inoperancia de ciertos departamentos administrativos, así como también por parte de los servidores públicos judiciales, que no permiten un cambio real dentro de ésta importante función del Estado.

Como ciudadanos y participes de la vida jurídica del Ecuador, hemos sido testigos que la Función Judicial durante años ha sido manejada por los estamentos políticos, lo que ha hecho que la justicia sea para unos cuantos y no para todos, es de ésta forma como lo ve el ciudadano común, y nos atreveríamos a decir, que actualmente persiste el mismo, ya que conocedores de los procesos de 
selección por mérito y oposición, sobre todo en lo que respecta a jueces, juezas secretarios/as, ayudantes judiciales, y operadores de justicia en general, se llevan a cabo muy cierto, aunque de manera lenta, en algunos casos se acierta, pero en otros no ha dado resultado, tal vez por la misma lentitud en el proceso de selección, hace que se vuelva una larga espera, que al final lleva a la misma situación, el mal continúa, la solución, volver a llamar a concurso, esto lo hemos visto en los últimos años, que se han cambiado a varios jueces/zas, por continuar con el sistema caduco y el retraso en el despacho de las causas.

Ante ésta grave situación es necesario crear conciencia de que debemos involucrarnos en el cambio del sistema judicial y no ser meros espectadores acostumbrados a ver las falencias del sistema y no hacer nada por cambiar ésta situación.

\section{Ventajas de la aplicación de los principios de inmediación y concentración en el proceso civil.}

Al implementarse una correcta y acertada aplicación de los principios de inmediación y concentración en el proceso civil oral ecuatoriano, encontraríamos varias ventajas que beneficiaría, a las partes involucradas en el proceso, así como también a los organismos estatales y a quienes están facultados a impartir justicia. Entre estas ventajas que hemos identificado las siguientes:

Como un claro ejemplo de la implementación del principio de concentración en el proceso citamos el siguiente: La audiencia única de pruebas, en que se presentan documentos, declaran los testigos, informan los peritos, se practican las confesiones judiciales. Como podemos ver en éste ejemplo, es una ventaja que en una sola audiencia se evacúen varias diligencias, puesto que esto elimina la tramitología del proceso escrito, que ha perdurado por varios años en el país, ocasionando un desgaste innecesario de recursos y tiempo. 
Gissela A. Cevallos-Sánchez; Zoila F. Alvarado-Moncada; Rene W. Astudillo-Orellana

Otra de las ventajas que se identifican a través de la inmediación es la aproximación del juez a las partes, lo que lo vuelve parte directa del mismo, y ya no un mero espectador, a partir de otros, es decir, sabemos que en el proceso escrito el juez sólo se limita a leer lo que le informan las partes, lo que no le permite tener una visión más objetiva de la causa puesta a su conocimiento.

Permite la intervención directa del juez en relación a las partes, a través de las preguntas que se les efectúen, de manera que se forme una convicción de los hechos por sí mismo, puesto que podrá observar y escuchar a las partes, denotando su tono de voz, expresiones, etc., lo que lo puede a formar su propio juicio.

En relación a las pruebas, en el nuevo sistema le permite al juez asistir a la práctica de valoración de las mismas, a través de ello puede formarse una mejor convicción de los hechos.

El principio de concentración se define como la posibilidad de ejecutar la máxima actividad del procedimiento en la fase oral.

Todo el material obtenido en la investigación se concentra en el juicio oral, a fin de que la actividad probatoria se desarrolle en una audiencia única y en el menor número de sesiones.Este principio le comunica rapidez al proceso y sitúa al juez en una mejor posición para fallar, también comprende a los incidentes y a las resoluciones interlocutorias.

\section{Conclusiones.}

Una vez que hemos finalizado nuestra investigación, podemos esbozar las conclusiones a las que hemos llegado, en relación al tema planteado, así tenemos:

Existe la necesidad imperiosa de una implementación efectiva y real de los principios constitucionales de inmediación y concentración, dentro del sistema de justicia en el país. 
Los procesos en materia civil no ven aun la luz de la celeridad, dentro de los trámites, lo que lógicamente ocasiona que las causas se retrasen, como consecuencia de la excesiva papelería en las diligencias, que aún persiste.

El sistema judicial, según la norma constitucional no impone la oralidad en todos los juicios, lo que causa el malestar en los ciudadanos que acuden ávidos de justicia.

Los servidores judiciales requieren de actualización de conocimientos en función de las normas legales vigentes, para las materias no penales, lo que les permitirá efectuar su trabajo de una forma ágil y expedita, y de esta manera brindar una mejor atención al usuario

\section{Bibliografía.}

MORELLO, A.. El Proceso Civil Moderno. (Buenos Aires, 2014) Editorial Platense.

RIOJA, Bermudez, A. El Nuevo Proceso Civil Peruano. (Arequipa Perú, 2011) Editorial Adrus.

VESCOVI, E. Teoria General del Proceso. (Texas, 1999) Editorial Temis.

ZAVALA, Baquerizo, Jorge. Tratado de derecho Procesal Penal. (Guayaquil-Ecuador, 2001) Juridica Edino.

Cabanellas de las Cuevas, G. Diccionario Jurídico Elemental . (2014) Heliasta.

Diccionario. Diccionario Enciclopédico . (Guayaquil, Guayas, Ecuador 2010). Editorial Océano.

Código Orgánico de la Función Judicial. Código Orgánico de la Función Judicial . (Guayaquil, Guayas, Ecuador, 2009) Ediciones Legales.

Constitución de la República . Art. 76. № 7. Literales a,b,c,e,y g . (Guayaquil, Guayas, Ecuador, 2008). Ediciones Legales.

http://definicionlegal.blogspot.com/2011/05/moral.html. (2014). Obtenido de http://definicionlegal.blogspot.com/2011/05/moral.html: http://definicionlegal.blogspot.com

http://www.enciclopedia-juridica.biz14.com/d/da\%C3\%B1o/da\%C3\%B1o.htm. (2014). Recuperado el noviembre de 2016, de http://www.enciclopedia-juridica.biz14.com/d/da\%C3\%

https://www.diccionariojuridico.mx/index.php?pag=vertermino\&id=150. (s.f.). Recuperado el $\begin{array}{lll}\text { octubre } & \text { de } & 2016,\end{array}$ https://www.diccionariojuridico.mx/index.php?pag=vertermino\&id=150: ht 
Gissela A. Cevallos-Sánchez; Zoila F. Alvarado-Moncada; Rene W. Astudillo-Orellana

Jesus

Maria

Gonzalez

Garcia.

(2012).

http://diccionario.pradpi.org/inicio/index.php/terminos_pub/view/33. Recuperado el 10 de 2016

Macedonio Hernandez, C. (s.f.). http://www.derecho.uady.mx/tohil/rev25/origenyevolucion.pdf.

Recuperado el 10 de noviembre de 2016 